\title{
Digital Dermatoscopy Method for Human Skin Roughness Analysis
}

\author{
Suprijanto $^{1}$, V. Nadhira ${ }^{1}$, Dyah A. Lestari ${ }^{1}$, E. Juliastuti ${ }^{1} \&$ Sasanti T. Darijanto ${ }^{2}$ \\ ${ }^{1}$ Instrumentation and Control Research Group, Faculty of Industrial Technology \\ ${ }^{2}$ Farmasetika Research Group, School of Pharmacy \\ Institute Technology of Bandung, Bandung Indonesia \\ Email: supri@tf.itb.ac.id
}

\begin{abstract}
In this study we propose a digital dermatoscopy method to measure the human skin roughness. By using this method we eliminate the use of silicon replica. Digital dermatoscopy consists of handheld digital microscope, image processing and information extraction of skin roughness level. To reduce the noise due to the variation of reflection factor on the skin we use median filter. Hence, by Fourier transform the skin texture is imaged in terms of 2D frequencyspatial distribution. Skin roughness is determined from its entropy, where the roughness level is proportional to the entropy. Three types of experiment have been performed by evaluating: (i) the skin replicas; (ii) young and elderly skin; and (iii) seven volunteers treated by anti wrinkle cosmetic in three weeks period. We find for the first and second experiment that our system did manage to quantify the roughness, while on the third experiment, six of seven volunteers, the roughness are succeeded to be identified.
\end{abstract}

Keywords: digital dermatoscopy; entropy; image processing; roughness level; skin imaging.

\section{Introduction}

Due to increasing environmental influences on the human skin, treatment and preservation of skin health using cosmetic is became one of the human demands. Several cosmetic industries offer a wide variety of skin care products $[1,2,3]$. One of cosmetic product types that used to make the consumer look younger is called anti wrinkle or anti-aging cream. It works by reducing visible wrinkles and expression lines. Considering the quality management, the promised effects of the anti wrinkle must be evaluated.

In conventional method, efficacy effect of anti wrinkle products can be performed by the skin expert. Therefore the evaluation results are often influenced by subjectivity factor. To obtain an objective result, a skin imaging technique is required to measure and analyze the human skin surface. The most common indirect imaging technique to investigate human skin topography is based on a measurement of a negative imprint of human skin micro-topography,

Received November $15^{\text {th }}, 2010$, Revised January $6^{\text {th }}, 2011,2^{\text {nd }}$ revision March $17^{\text {th }}, 2011$, Accepted for publication March $20^{\text {th }}, 2011$. 
made of fast hardening silicon mass or often called the skin replica-silicon [1,2]. The optical device such as optical profilometry or transmition profilometry is used to evaluate the wrinkle level based on skin replica. It has a limitation on the spatial resolution of the skin and its procedure on making the silicon skin replica.

As an alternative, a prototype digital dermatoscopy method is proposed as a direct measurement for human skin roughness. Digital dermatoscopy consists of handheld digital microscope, image processing and information extraction of skin roughness level [3]. This paper describes the current status of the research on digital dermatoscopy method for human skin roughness analysis. A commercial handheld digital microscope is used. The image processing and information extraction of skin roughness level, to determine the wrinkle skin level, based on investigated skin surface area are discussed.

\section{Literature Study}

\subsection{Human Skin Surface}

The human skin is responsible for many vital functions. It protects our internal body from the environment. The main layer of human skin is shown in Figure 1. The upper layer of epidermis is known as stratum corneum. The structure of the stratum corneum can be described as a "brick and mortar" type structure [4].

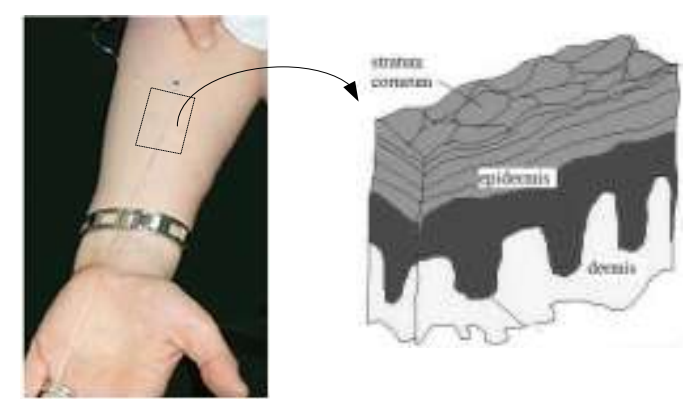

(a)

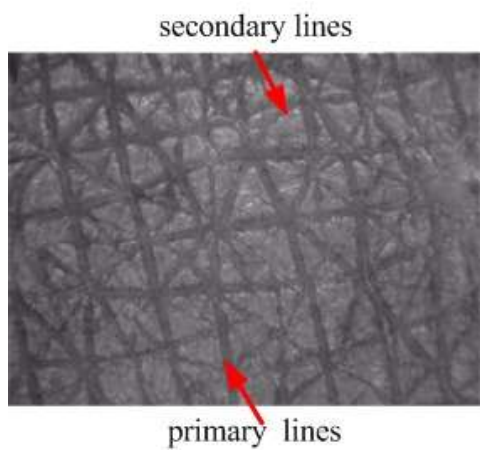

(b)

Figure 1 (a) The main layer of human skin (b) skin surface that show as intersecting primary and secondary lines.

The corneocyte is described as a brick. A corneocyte is a protein complex that is made of tiny threads of keratin in an organized matrix. The keratin can hold large amounts of water between its fibers. 
The stratum corneum contains about 12-16 layers of corneocytes and each corneocyte has a thickness of about 1 micrometer, depending on age, anatomical location and exposure to ultra violet radiation. The pattern of corneocytes distribution will be related to texture of the skin surface. The skin surface is intersected by primary and secondary lines (See Figure 1). The role of anti wrinkle cosmetic is reducing visible wrinkles and expression primary and secondary lines. The quantitative analysis of wrinkles level is frequently used in skin analyzer. Various techniques have been developed for skin imaging and in particular micro-topography which can be separated into optical, mechanical and laser. In the further section, overview about optical skin surface imaging is described.

\subsection{Optical Skin Surface Imaging Techniques}

Micro-skin topography can be imaged using digital dermatoscopy. Digital dermatoscopy consists of optical lens system to magnify profile of skin surface, CCD sensor to convert analog image to digital image and interface to a computer [4-6]. On the prototype of imaging system, the digital dermatoscopy with build-in luminance system is used. The block diagram of skin imaging using optical skin imaging is shown in Figure 2. Using this system, microtopography of surface skin is represented in the digital image. The intensity value of the image is a function of the reflected light on the topography of skin surface.

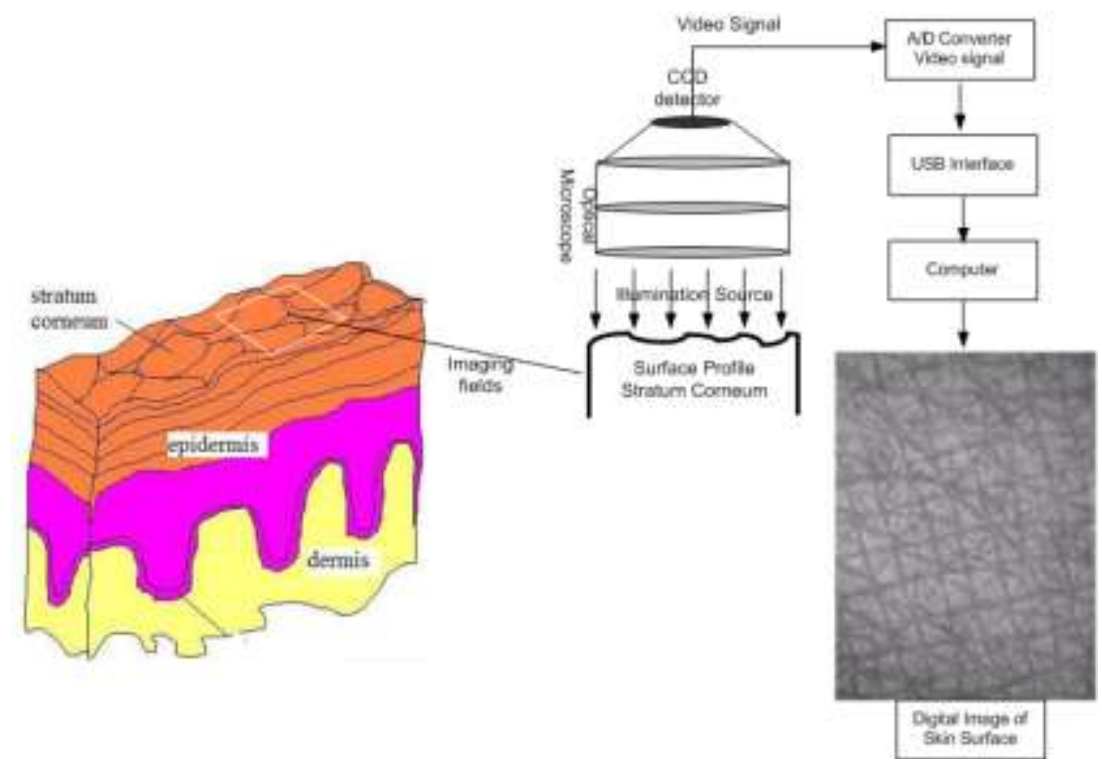

Figure 2 The prototype of skin imaging using optical skin imaging. 


\subsection{Image Processing}

\subsubsection{Digital Skin Images}

A digital image $a[m, n]$ described in a $2 \mathrm{D}$ discrete space is derived from an analog image $a(x, y)$ in a $2 \mathrm{D}$ continuous space through a sampling process. On the skin images, the value of $a(x, y)$ is related with the detection of reflected light which is depend on the depths and the angles of the topography of skin surface [6,7]. Converting from a continuous image $a(x, y)$ to its digital representation $a[m, n]$ requires the process of sampling. In the ideal sampling system $a(x, y)$ is convolved by an ideal 2D impulse train $\delta(\bullet, \bullet)[5,8]$, that modeled in Eq. 1:

$$
\begin{aligned}
a[m, n] & =a(x, y) \otimes \sum_{i=-\infty}^{+\infty} \sum_{n=-\infty}^{+\infty} \delta\left(x-m X_{o}, y-n Y_{o}\right) \\
& =\sum_{i=-\infty}^{+\infty} \sum_{n=-\infty}^{+\infty} a\left(m X_{o}, n Y_{o}\right) \delta\left(x-m X_{o}, y-n Y_{o}\right)
\end{aligned}
$$

where $X_{0}$ and $Y_{o}$ are the sampling distances or intervals square sampling implies that $X_{o}=Y_{o}$. With current technology, conversion from analog to digital image is done on integrated electronic system on the digital microscope and transmitted digital image to computer through USB interface.

\subsubsection{Smoothing Filter}

The contrast on the digital skin imaging is a function of the reflected light from the skin surface that likes a topographical map with plateaus and valleys. A Charge Couple Device (CCD) camera produces a type of noise, where photons produce electrons, which known as photoelectrons [7]. The source of noise on CCD is frequently limited by a noise amplifier; thermal noise that related with the chip temperature and exposure time; and photon noise which is limited by the photon production rate and the exposure time [6].

It is assumed that the skin topography varied slowly, so that the noise in terms of high frequency signal the skin image is filtered by a smoothing filter. The basic principle of smoothing filter is the convolution operation. The basic idea of its operation is defined a window of some finite size and shape then scanning across the image. The output pixel value is the weighted sum of the input pixels within the window where the weights are the values of the filter assigned to every pixel of the window itself. The window with its weights is called the convolution kernel $-\mathrm{h}[\mathrm{j}, \mathrm{k}]$. If $\mathrm{h}[\mathrm{j}, \mathrm{k}]$ is zero outside the window of size $J x K$ centered around the origin $\left\{\mathrm{j}=-\mathrm{J}_{\mathrm{o}},-\mathrm{J}_{\mathrm{o}}+1, \ldots \ldots,-1,0,1, \ldots \ldots \ldots, \mathrm{J}_{\mathrm{o}}-1, \mathrm{~J}_{\mathrm{o}} ; \mathrm{k}=-\mathrm{K}_{\mathrm{o}}\right.$,$\left.\mathrm{K}_{\mathrm{o}}+1, \ldots \ldots, 1,0,1, \ldots \ldots \ldots, \mathrm{K}_{\mathrm{o}}-1, \mathrm{~K}_{\mathrm{o}}\right\}$. Formally, the convolution operation is written as 


$$
c[m, n]=a[m, n] \otimes h[m, n]=\sum_{j=-J_{o}}^{J_{o}} \sum_{k=-K_{o}}^{K_{o}} h[j, k] a[m-j, n-k]
$$

Where $c[m, n]$ is a convolved image. There are two types of smoothing filter which can be distinguished, linear and non-linear algorithms. The examples of linear filtering algorithms are uniform or averaging filter and Gaussian filter. However, the filtered skin imaging, the smoothing filters with characteristic to preserve the edge of skin texture while suppressing the noise contamination is required. A type of the non-linear filters that covers these characteristics is a median filter.

The working principle of a median filter is by moving a window over an image (as in a convolution) and computing the output pixel as the median value of the brightness within the input window. If the window is $\mathrm{J} \times \mathrm{K}$ in size we can order the $\mathrm{J} \cdot \mathrm{K}$ pixels in brightness value from smallest to largest. If $\mathrm{J} \bullet \mathrm{K}$ is odd then the median will be the $(\mathrm{J} \cdot \mathrm{K}+1) / 2$ entry in the list of ordered brightness.

\subsubsection{Texture Analysis in the Spatial Frequency Domain}

Texture analysis is required to evaluate the wrinkles level of micro-topography [3,6-9]. An approach to analyze the texture is by generating the Fourier transform of the image and then to analyze further the transformed data in some way to obtain a set of measurements $[3,8,9]$.

The basic principle of the 2D digital Fourier transform (2D-DFT) is to transform the spatial domain images a[m,n] to the frequency domain $\mathrm{F}\left(\Omega_{\mathrm{m}}, \Psi_{\mathrm{n}}\right)$,

$$
F\left(\Omega_{m}, \Psi_{n}\right)=\sum_{m=-\infty}^{+\infty} \sum_{n=\infty}^{+\infty} a[m, n] e^{-j(\Omega m+\Psi n)}=F[a[m, n]]
$$

The notation $\mathrm{F}\left(\Omega_{\mathrm{m}}, \Psi_{\mathrm{n}}\right)$ is the DFT of $a[m, n]$, where $\Omega$ and $\Psi$ are the coordinates in the inverse space of FT and $a[m, n]$ is the image of micro topography. The function $\mathrm{F}\left(\Omega_{\mathrm{m}}, \Psi_{\mathrm{n}}\right)$ is a complex function, therefore Equation 3 can be written as

$$
F\left(\Omega_{n}, \Psi_{m}\right)=\left|F\left(\Omega_{n}, \Psi_{m}\right)\right| e^{-j \phi\left(\Omega_{n}, \Psi_{m}\right)}
$$

where the first part $\left|\mathrm{F}\left(\Omega_{\mathrm{m}}, \Psi_{\mathrm{n}}\right)\right|$ is the modulus while the second part with the exponential $e^{-\mathrm{j} \varphi(\Omega, \psi)}$ is the phase. Furthermore, the transformed data is normalized by the sum of the squared values of each magnitude component (excepting the zero-frequency components, those for $\mathrm{m}=0$ and $\mathrm{n}=0$ ). The normalized Fourier coefficients $\left(N F P_{m, n}\right)$ is given by 


$$
N F P_{n, m}=\frac{\left|F\left(\Omega_{n}, \Psi_{m}\right)\right|}{\sqrt{\sum_{(m \neq o),(n \neq o)}\left|F\left(\Omega_{n}, \Psi_{m}\right)\right|^{2}}}
$$

where $N F P_{m, n}$ is invariant to the linear shifting of illumination. On the NFP map, the spatial frequency is proportional to the radius relative to the center (see on Figure 2).

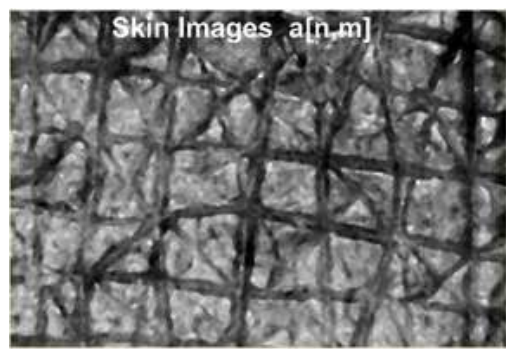

(a)

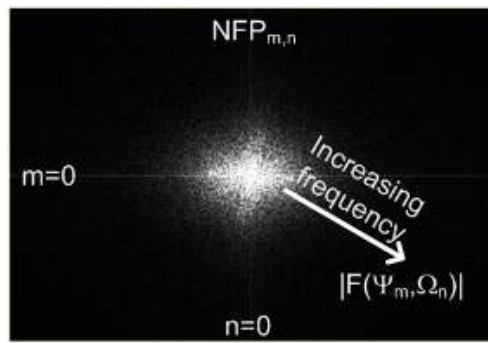

(b)

Figure 3 Digital skin image that acquired using direct method (a) and (b) normalized Fourier coefficients (NFP).

The skin roughness level is hypothetically related to the pattern of the visible wrinkles and expression lines on the skin images (See Figure 1-b). The NFP map pattern shows that the increasing of the spreading value of high frequency increase the degree of NFP randomness. A statistical measure of randomness NFP is represented by the entropy value $(h)$,

$$
h=\sum_{n-1}^{N} \sum_{m=1}^{M} N F P_{n, m} \log \left(N F P_{n, m}\right)
$$

The increasing of $h$ value describes the increasing levels of skin wrinkles that related with increasing level of skin roughness.

\section{Materials and Experiment Design}

We use DYNOLAB DinoLite Snap AM-311ST as the handheld digital microscope with build-in luminance system. To evaluate the performance of the image processing scheme for skin analyzer, two types of experiments have been done.

\subsection{The First Experiment Design}

The objective of the first experiment design is to evaluate the performance of digital dermatoscopy to analyze wrinkles level of silicone skin replicas. Two 
silicon skin replicas are taken from skin sites before the anti wrinkle cosmetic and after 40 days usage.

Based on silicon skin replica, the optical skin imaging is used to obtain the digital images. The intensity distribution depends on the reflected light which is a function of the depths and the angles of the wrinkles. Block diagram of the first experiment design is shown in Figure 3.

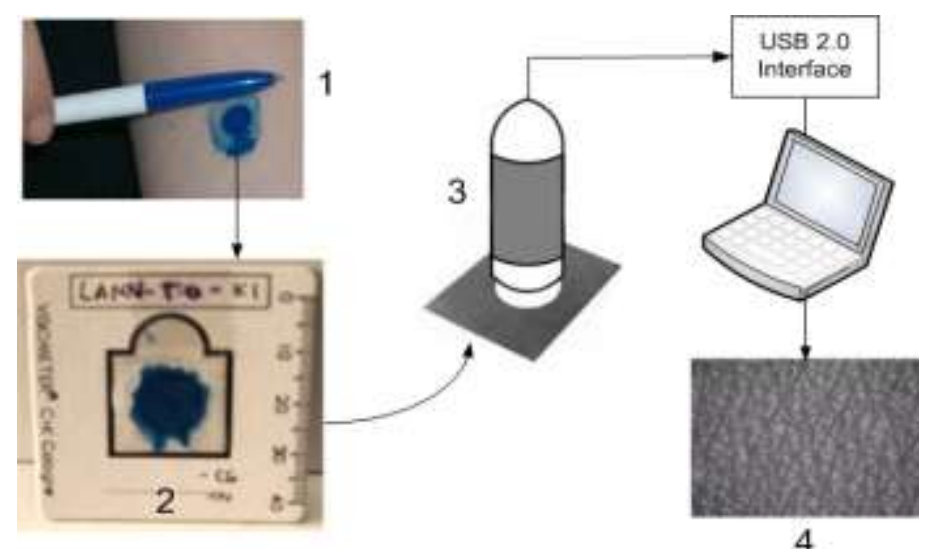

Figure 4 Optical skin imaging based on silicone skin replica. (1) Mixed silicon drop on the skin surface that bounded using the plastic ring (2) The silicon-skin replica that located in the special carrier (3) Skin replica imaged using digital dermatoscopy to obtain (4) the digital image.

\subsection{Second Experiment Design}

The objective of the second experiment design is to evaluate the performance of the digital dermatoscopy to analyze wrinkles level on the skin surface. The experiment is done by taking the image before the use of the anti wrinkle cosmetic and after. During the imaging process, the digital microscope is positioned close to the target area and illuminated directly through the lens. The luminous environment is uniform so that all of the shooting sessions and both images are obtained under the same conditions. Images are collected at 50x magnification and downloaded to a personal computer through USB interface. The block diagram of the second experiment design is shown in Figure 4. 


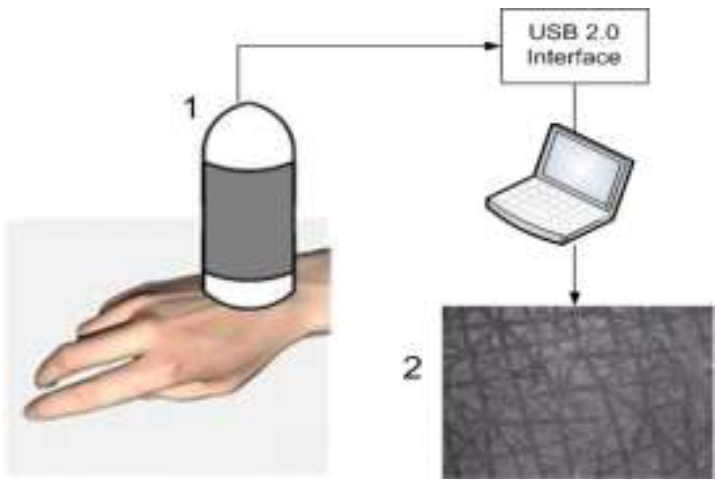

Figure 5 The direct and in-situ skin imaging using optical skin imaging. (1) the handheld digital microscope with build-in luminance system and (2) obtained digital skin image that represent the micro-skin topography.

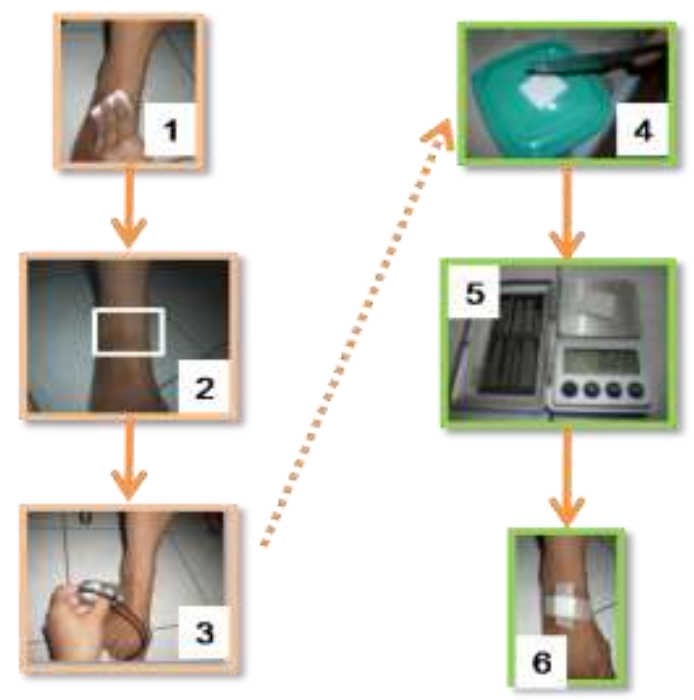

Figure 6 The step procedures of skin preparation and acquired skin images. (1) the image of skin surface is cleaned using milk cleanser and face toner in order to improve the image quality; (2) determine the wrinkle area; (3) image retrieval using CCD microscope; (4) and (5) sterile gauze is lubricated with 2 grams antiwrinkle; (6) sterile gauze with anti-wrinkle is placed on the experimental area skin surface. The above procedures are repeated everyday for 3 weeks.

From the second experiment design, two experiment setups have been done. The first setup is used to analyze the wrinkle levels of skin image between the inner forearm of skin of the volunteers of age 12 and 40. The second experiment setup is used to analyze the skin wrinkle alteration due to the use of an anti wrinkle cosmetic for 3 weeks on the forearm skin of 7 volunteers. 
The skin treatment is supervised by a cosmetic expert so that the treatment is done under the same condition. The experiment procedures are illustrated in Figure 5 (indicated by number).

\subsection{Processing Steps to Skin Analyzer}

All collected images are referred as raw skin image, with size 640x480 pixels. The feature of skin analyzer is implemented in 3D skin surface, surface profile and the texture description of micro-topography. The step of processing is shown in Figure 6.

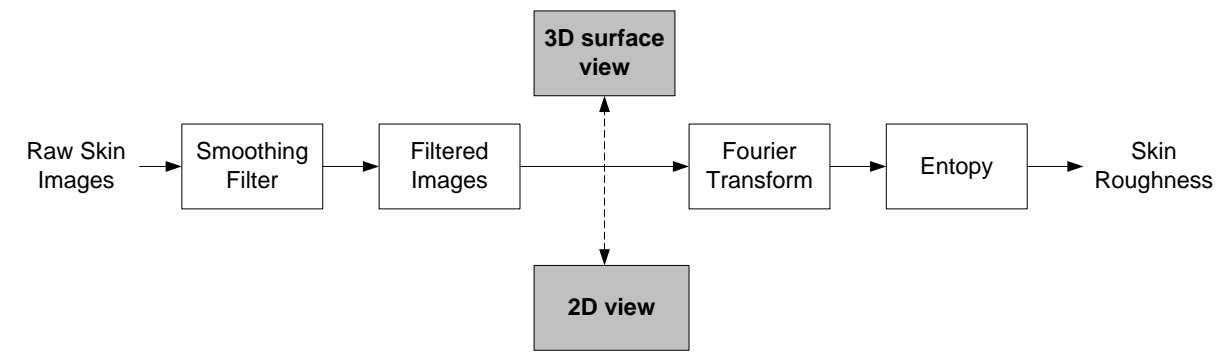

Figure 7 The processing steps of skin analyzer.

Raw skin image is processed to minimize the noise so that it can represent the topography of the skin. The procedure is done as follows: (1) the image selection; (2) smoothing using median filter, (3) transforming the filtered image from spatial domain into frequency domain and to obtain the NFP map; (4) to calculate the randomness degree of NFP map using entropy; and (5) to determine the roughness level of the skin surface from the entropy value.

\section{Results}

\subsection{Results of First Experiment Design}

The filtered images of silicon skin replicas are shown in Figure 7. The intersection lines on the images represent the topography of the skin surface. The skin topography of the skin replica is imaged in 3D (Figure 7-a) as shown in Figure 8. The smoothing filter effect is significantly increasing the quality of the topography map. 


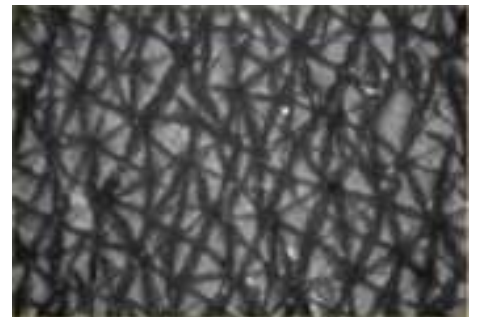

(a)

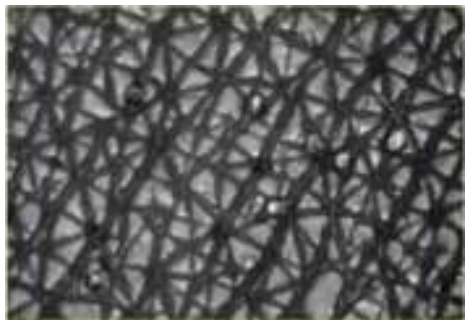

(b)

Figure 8 Filtered image from silicon skin replica before (a) and 40 days after anti wrinkle treatment (b).

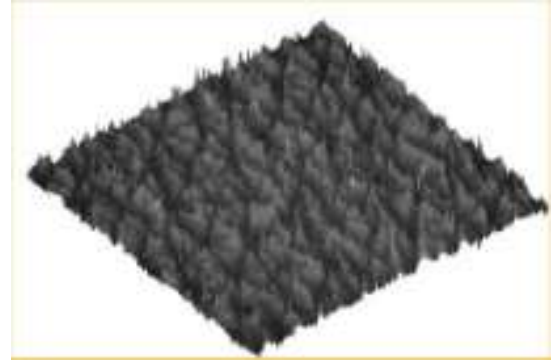

(a)

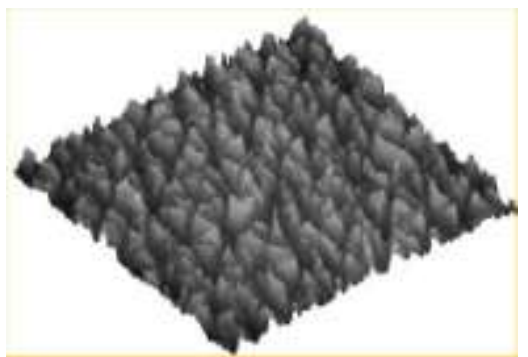

(b)

Figure 9 3D surface view of skin topography of Figure 6-a. without (a) and with (b) smoothing filter.

To determine the texture description, Fourier transform is performed on both skin replica images. The magnitude of Fourier transform coefficients of both images are shown in Figure 9.
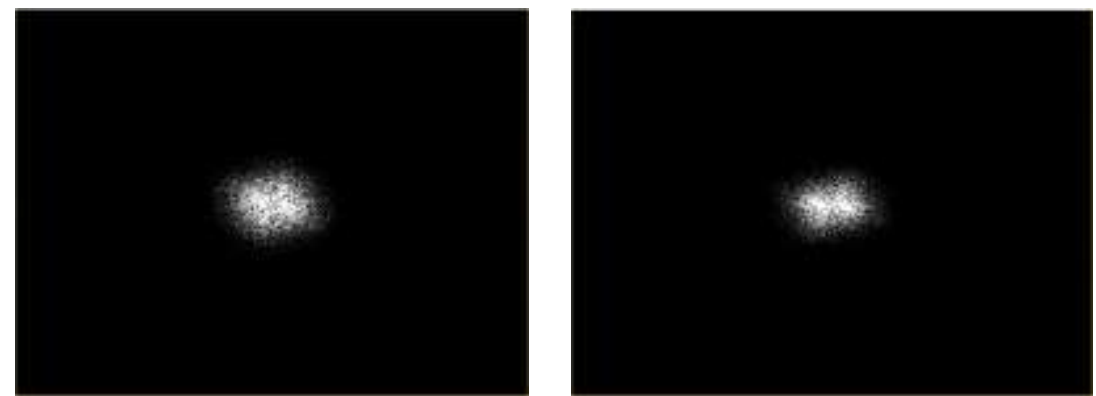

Figure 10 The NFP map of silicon skin replica before (a) and 40 days after anti wrinkle treatment $(b)$.

The value of the entropy is calculated for both images show that from before and after treatment the images have the entropy value of $h=4.34$ and $h=3.03$. It 
is shown from the results that the effect of anti wrinkle treatment will reduce the roughness level on the skin surface.

\subsection{Results of Second Experiment Design}

\subsubsection{Results of First Setup}

The filtered images of the inner forearm skin of the volunteers of age 12 and 40 year-old are shown in Figure 10. The profile different of skin topography of both image samples is shown in Figure 11 (blue line related to the age of 12 and red line related to the age of 40). The skin profile topography shows that the minimum and maximum profile changes are bigger on the older skin type.

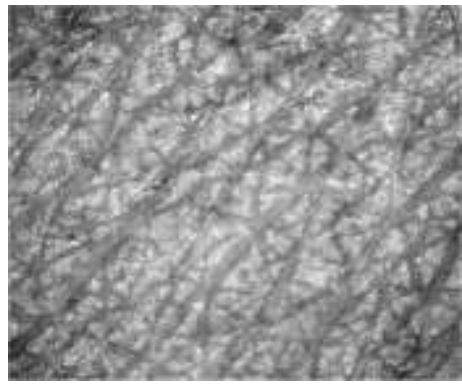

(a)

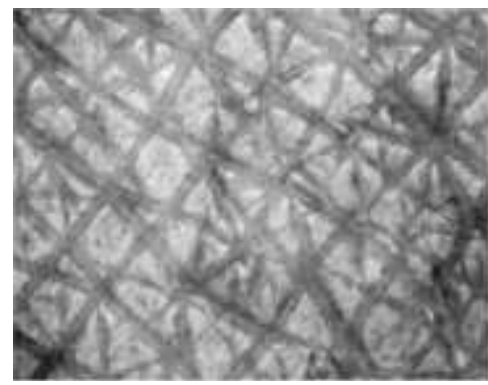

(b)

Figure 11 The filtered skin image of the inner forearm from: (a) female 12 year and (b) male 40 year.

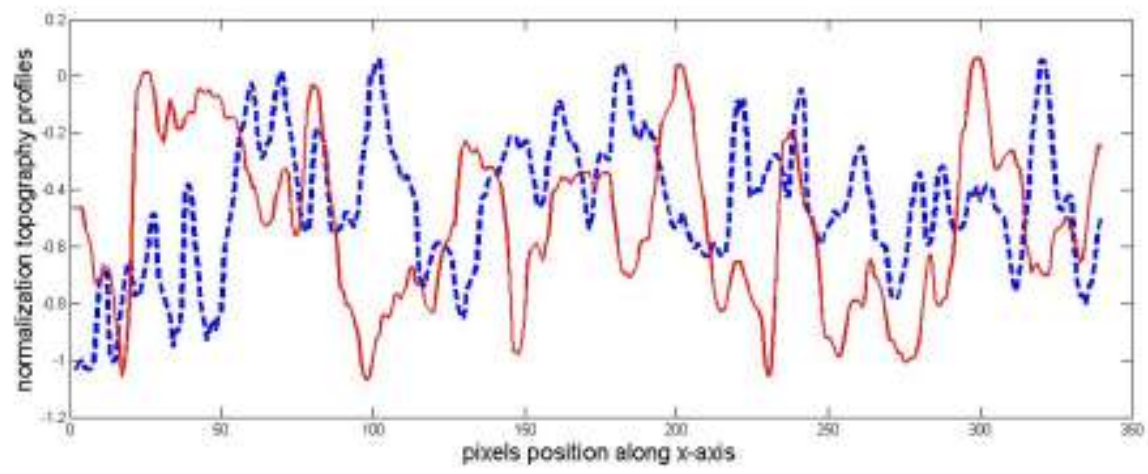

Figure 12 Profile of skin topography (dashed line related with age 12 year and solid line related with age 40 year).

The texture descriptions from both images are determined using the Fourier transform. The NFP map of both images is shown in Figure 12. 


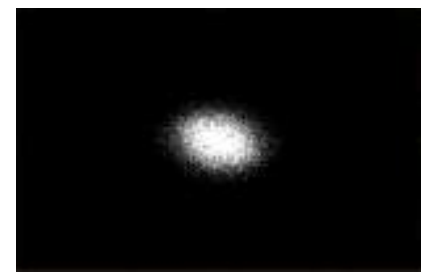

(a)

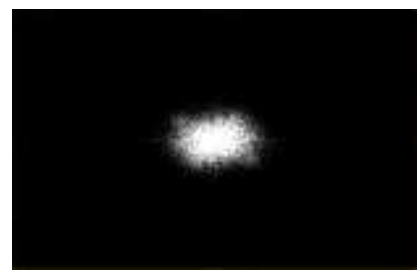

(b)

Figure 13 The NFP map of the inner forearm for: (a) female with age 12 year and (b) male with age 40 year.

The NFP map pattern is more symmetry on the younger skin. The calculated entropy of young skin is $h=1.20$, while for older skin is $h=1.42$. The results indicate the increasing roughness level is proportional to the entropy value. It is directly correspond with the subjective criterion of the skin roughness.

\subsubsection{Results of Second Setup}

The examples of the skin images of subject 3 are shown in Figure 13. The skin image processing evaluation is done by following the steps given in Figure 6 . We set the T0 image as the entropy reference. Therefore, the relative entropy of $\mathrm{T} 0$ image is set to be a $100 \%$. The relative entropy of $\mathrm{T} 1$ and $\mathrm{T} 2$ images are calculated relative to the entropy reference value.

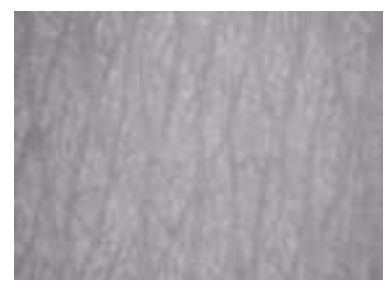

$\mathrm{T}_{\mathrm{o}}$

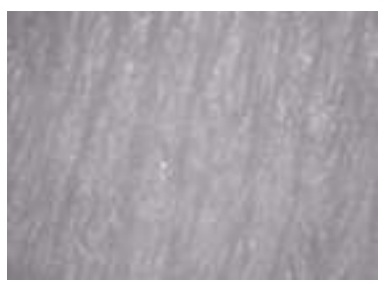

$\mathrm{T}_{1}$

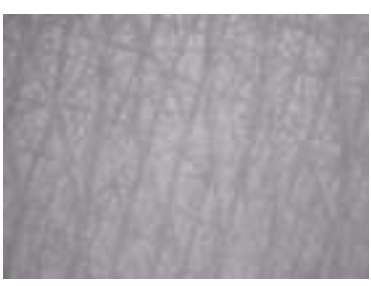

$\mathrm{T}_{2}$

Figure 14 The examples skin images from subject 3 that treatment antiwrinkle (using procedure shown in Figure 5) during 3 weeks. Image that indicated by $\mathrm{T}_{\mathrm{o}}, \mathrm{T}_{1}$ and $\mathrm{T}_{2}$ were acquired on the $1^{\text {st }}, 2^{\text {th }}$, and $3^{\text {th }}$ weeks. (Note image on $1^{\text {st }}$ week was acquired before anti wrinkle treatment).

The secondary lines of visible wrinkles are reduced by treating the skin with the anti wrinkle cosmetic as shown in Figure 13. Therefore, the statistical interpretation of the 2D randomness pattern of NFP tends to decrease. Hence, the value of entropy tends to decrease on $\mathrm{T} 1$ and $\mathrm{T} 2$ images relative to $\mathrm{T} 0$ image.

The results are summarized in Table 1. The trend of slope from relative entropy is determined using linear regression. The negative slope indicates the 
decreasing relative entropy which is proportional to the decreasing of the roughness skin level.

Table 1 Evaluation Results of Wrinkle Level due to anti-wrinle treatments from 7 subjects during three weeks.

\begin{tabular}{|c|c|c|c|}
\hline Subject & $\begin{array}{l}\text { Interval Time for } \\
\text { acquired images }\end{array}$ & $\begin{array}{l}\text { Relative } \\
\text { entropy }\end{array}$ & Trend of Slope \\
\hline \multirow{3}{*}{ Volunteer 1} & T0 & 100 & \multirow{3}{*}{$\begin{array}{c}-3.47 \\
(\text { down })\end{array}$} \\
\hline & $\mathrm{T} 1$ & 76.09 & \\
\hline & $\mathrm{T} 2$ & 93.06 & \\
\hline \multirow{3}{*}{ Volunteer 2} & T0 & 100 & \multirow{3}{*}{$\begin{array}{l}-11.03 \\
\text { (down) }\end{array}$} \\
\hline & $\mathrm{T} 1$ & 66.67 & \\
\hline & $\mathrm{T} 2$ & 77.94 & \\
\hline \multirow{3}{*}{ Volunteer 3} & T0 & 100 & \multirow{3}{*}{$\begin{array}{l}5.02 \\
\text { (up) }\end{array}$} \\
\hline & $\mathrm{T} 1$ & 99.39 & \\
\hline & $\mathrm{T} 2$ & 110.04 & \\
\hline \multirow{3}{*}{ Volunteer 4} & T0 & 100 & \multirow{3}{*}{$\begin{array}{l}-1.94 \\
(\text { down })\end{array}$} \\
\hline & $\mathrm{T} 1$ & 85.25 & \\
\hline & $\mathrm{T} 2$ & 96.12 & \\
\hline \multirow{3}{*}{ Volunteer 5} & T0 & 100 & \multirow{3}{*}{$\begin{array}{l}-11.93 \\
(\text { down })\end{array}$} \\
\hline & $\mathrm{T} 1$ & 80.16 & \\
\hline & $\mathrm{T} 2$ & 76.12 & \\
\hline \multirow{3}{*}{ Volunteer 6} & T0 & 100 & \multirow{3}{*}{$\begin{array}{l}-24.27 \\
(\text { down })\end{array}$} \\
\hline & $\mathrm{T} 1$ & 124.16 & \\
\hline & $\mathrm{T} 2$ & 51.45 & \\
\hline \multirow{3}{*}{ Volunteer 7} & T0 & 100 & \multirow{3}{*}{$\begin{array}{c}-3.59 \\
(\text { down })\end{array}$} \\
\hline & $\mathrm{T} 1$ & 86.89 & \\
\hline & $\mathrm{T} 2$ & 92.83 & \\
\hline
\end{tabular}

\section{Conclusions and Future Work}

This paper demonstrates the alternative direct skin roughness analysis using a digital dermatoscopy method. Three types of experiment have been performed 
by evaluating: (i) the skin replicas; (ii) young and elderly skin; and (iii) seven volunteers treated by anti wrinkle cosmetic in three weeks period. We find for the first and second experiment that our system did manage to quantify the roughness. On the third experiment, six of seven volunteers, the roughness are succeeded to be identified since the anti wrinkle treatment has no effect on a particular skin type.

The quantification is obtained by calculating the NFP map. The map profiles indicate the skin roughness level is proportional and tends to follow the negative slope of the relative entropy. One of the advantages of the digital dermatoscopy method is the time interval needed to quantify the roughness level changing can be reduced compared to the skin replica's method where for skin replica it takes about three months, while on digital dermatoscopy is about three weeks.

For the future research is to deal with the skin curvature and light reflection factor on the skin surface. By applying a polarization filter [5] and improving the illumination technique are the two possible solutions to improve the performance of the digital dermatoscopy system.

\section{References}

[1] Kollias, Nikiforos, Skin Documentation with Multimodal Imaging or Integrated Imaging Approaches, in Bioengineering of The Skin, Vol. II, Klaus-P Wilhem, et al. Ed. New York: Informa Healthcare USA, Inc, pp. 221-246, 2007.

[2] Murphy, R. \& Contton, D.W.K., et al., Computer-Assisted Image Analysis of Skin Surface Replicas, British Journal of Dermatology, 124(6), 571-575, 2006.

[3] Suprijanto, Ayu, D., Nadira, V. \& Darijanto, S.T., Development of Image Processing for digital Dermatoscopy, Proceeding International Conference on Instrumentation, Communications, Information Technology, and Biomedical Engineering (ICICI-BME), 2009

[4] El Gammal, Claudia, El Gammal, Stephan \& Albert M Klingman, Anatomy of the Skin Surface, in Bioengineering of The Skin, II, Klaus-P. Wilhelm, et al. Ed. New York: Informa Healthcare USA, Inc, pp. 1-17, 2007.

[5] Bae, Eui Jong, et al., A Quantitative Assessment of the Human Skin Surface Using Polarized Light Digital Photography and its Dermatologic Significance, Skin Research and Technology, January 2010.

[6] Young, I.T., et al., Fundamentals of Image Processing. Faculty of Applied Physics, Pattern Recognition Group, Delft University of Technology, 2000. 
[7] Randen, T. \& Husoy, J.H., Filtering for Texture Classification: A Comparative Study, IEEE Transactions on Pattern Analysis and Machine Intelligence, 291-310, April 1999.

[8] Tanaka, Hiromasa, et al., Quantitative Evaluation of Elderly Skin Based on Digital Image Analysis, Blackwell Munksgaard Journal Compilation, 14, pp. 192-199, May 2007.

[9] Setaro, Michele \& Sparavigna, Adele, Irregularity Skin Index (ISI): A Tool to Evaluate Skin Surface Texture, Skin Research and Technology, 7(3), 159-163, 2001. 